\title{
MULTI-HYPERSUBSTITUTIONS AND COLORED SOLID VARIETIES
}

\author{
K. DENECKE, J. KOPPITZ, SL. SHTRAKOV
}

\begin{abstract}
Hypersubstitutions are mappings which map operation symbols to terms. Terms can be visualized by trees. Hypersubstitutions can be extended to mappings defined on sets of trees. The nodes of the trees, describing terms, are labelled by operation symbols and by colors, i.e. certain positive integers. We are interested in mappings which map differently colored operation symbols to different terms. In this paper we extend the theory of hypersubstitutions and solid varieties to multi-hypersubstitutions and colored solid varieties. We develop the interconnections between such colored terms and multi-hypersubstitutions and the equational theory of Universal Algebra. The collection of all varieties of a given type forms a complete lattice which is very complex and difficult to study; multi-hypersubstitutions and colored solid varieties offer a new method to study complete sublattices of this lattice.
\end{abstract}

\section{INTRODUCTION}

Let $X=\left\{x_{1}, \ldots, x_{n}, \ldots\right\}$ be a countably infinite set of variables, let $X_{n}=\left\{x_{1}, \ldots, x_{n}\right\}$ be a finite set and let $\left(f_{i}\right)_{i \in I}$ be a set of operation symbols where $f_{i}$ is $n_{i}-$ ary. The sequence $\tau:=\left(n_{i}\right)_{i \in I}$ is called a type. In the usual way from variables and operation symbols we build up the set $W_{\tau}(X)$ of all terms of type $\tau$. An algebra $\mathcal{A}=\left(A ;\left(f_{i}^{\mathcal{A}}\right)_{i \in I}\right)$ of type $\tau$ is a pair consisting of a set $A$ and an indexed set of operations defined on $A$. We denote by $\operatorname{Alg}(\tau)$ the class of all algebras of type $\tau$. If $s, t \in W_{\tau}(X)$, then the pair $s \approx t$ is called an identity in the algebra $\mathcal{A}$, if the term operations $s^{\mathcal{A}}$ and $t^{\mathcal{A}}$ induced by the terms $s$ and $t$ on the algebra $\mathcal{A}$ are equal. In this case we write $\mathcal{A} \models s \approx t$. The binary relation $\models \subseteq A l g(\tau) \times W_{\tau}(X)^{2}$ gives rise to a Galois connection $(I d, M o d)$ between the power sets of $\operatorname{Alg}(\tau)$ and $W_{\tau}(X)^{2}$, where $I d$ and Mod are defined for $K \subseteq A l g(\tau)$ and $\Sigma \subseteq W_{\tau}(X)^{2}$ by

$$
I d K:=\{s \approx t \mid \forall \mathcal{A} \in K(\mathcal{A} \models s \approx t)\},
$$

2000 Mathematics Subject Classification. Primary: 08A15; Secondary: 08A25.

Key words and phrases. Coloration of terms, Multi-hypersubstitutions, Colored solid varieties. 


$$
\operatorname{Mod} \Sigma:=\{\mathcal{A} \mid \forall s \approx t \in \Sigma(\mathcal{A} \models s \approx t)\} .
$$

As a Galois connection, (Id, Mod) has the properties:

$$
\begin{gathered}
\Sigma_{1} \subseteq \Sigma_{2} \Rightarrow \operatorname{Mod} \Sigma_{2} \subseteq \operatorname{Mod} \Sigma_{1}, K_{1} \subseteq K_{2} \Rightarrow I d K_{2} \subseteq I d K_{1}, \\
\Sigma \subseteq \operatorname{IdMod} \Sigma, K \subseteq \operatorname{ModIdK} .
\end{gathered}
$$

From these properties of the Galois connection (Id, Mod) we obtain that the fix points with respect to the closure operators

$$
\text { IdMod }: \mathcal{P}\left(W_{\tau}(X)^{2}\right) \rightarrow \mathcal{P}\left(W_{\tau}(X)^{2}\right)
$$

and

$$
\operatorname{ModId}: \mathcal{P}(A l g(\tau)) \rightarrow \mathcal{P}(A l g(\tau))
$$

form complete lattices

$$
\begin{aligned}
\mathcal{L}(\tau) & :=\{K \mid K \subseteq \operatorname{Alg}(\tau) \text { and } \operatorname{Mod} I d K=K\} \\
\mathcal{E}(\tau) & :=\left\{\Sigma \mid \Sigma \subseteq W_{\tau}(X)^{2} \text { and } \operatorname{IdMod} \Sigma=\Sigma\right\}
\end{aligned}
$$

of all varieties of type $\tau$ and of all equational theories of type $\tau$. These lattices are dually isomorphic.

Our next goal is to introduce two new closure operators on our sets $A l g(\tau)$ and $W_{\tau}(X)^{2}$ which give us complete sublattices of our two lattices $\mathcal{L}(\tau)$ and $\mathcal{E}(\tau)$. The new operators are based on the concept of hypersatisfaction of an identity by a variety. We begin with the definition of a hypersubstitution. A complete study of hypersubstitutions may be found in 2 .

A hypersubstitution of type $\tau$ is a map which associates to every operation symbol $f_{i}$ a term $\sigma\left(f_{i}\right)$ of type $\tau$, of the same arity as $f_{i}$. Any hypersubstitution $\sigma$ can be uniquely extended to a map $\hat{\sigma}$ on the set $W_{\tau}(X)$ of all terms of type $\tau$ as follows:

(i) If $t=x_{j}$ for some $j \geq 1$, then $\hat{\sigma}[t]=x_{j}$;

(ii) if $t=f_{i}\left(t_{1}, \ldots, t_{n_{i}}\right)$ for some $n_{i}$-ary operation symbol $f_{i}$ and some terms $t_{1}, \ldots, t_{n_{i}}$, then $\hat{\sigma}[t]=\sigma\left(f_{i}\right)\left(\hat{\sigma}\left[t_{1}\right], \ldots, \hat{\sigma}\left[t_{n_{i}}\right]\right)$.

Here the right side of (ii) means the composition of the term $\sigma\left(f_{i}\right)$ and the terms $\hat{\sigma}\left[t_{1}\right], \ldots, \hat{\sigma}\left[t_{n_{i}}\right]$.

We can define a binary operation $\circ_{h}$ on the set $\operatorname{Hyp}(\tau)$ of all hypersubstitutions of type $\tau$, by taking $\sigma_{1} \circ_{h} \sigma_{2}$ to be the hypersubstitution which maps each fundamental operation symbol $f_{i}$ to the term $\hat{\sigma}_{1}\left[\sigma_{2}\left(f_{i}\right)\right]$. That is,

$$
\sigma_{1} \circ_{h} \sigma_{2}:=\hat{\sigma}_{1} \circ \sigma_{2},
$$


where $\circ$ denotes the ordinary composition of functions. The operation $\circ_{h}$ is associative. The identity hypersubstitution $\sigma_{i d}$ which maps every $f_{i}$ to $f_{i}\left(x_{1}, \ldots, x_{n_{i}}\right)$ is an identity element for this operation. Then $\mathcal{H} y p(\tau):=\left(\operatorname{Hyp}(\tau) ; \circ_{h}, \sigma_{i d}\right)$ is a monoid.

Definition 1.1. Let $\mathcal{M}$ be any submonoid of $\mathcal{H} y p(\tau)$. An algebra $\mathcal{A}$ is said to $M$-hypersatisfy an identity $u \approx v$ if for every hypersubstitution $\sigma \in M$, the identity $\hat{\sigma}[u] \approx \hat{\sigma}[v]$ holds in $\mathcal{A}$. In this case we say that the identity $u \approx v$ is an $M$-hyperidentity of $\mathcal{A}$ and we write $\mathcal{A} \underset{M-h y p}{\models}$ $u \approx v$. For $M=\operatorname{Hyp}(\tau)$ we write $\mathcal{A} \underset{h y p}{\models} u \approx v$.

An identity is called an $M$-hyperidentity of a variety $V$ if it holds as an $M$-hyperidentity in every algebra in $V$. A variety $V$ is called $M$ solid if every identity of $V$ is an $M$-hyperidentity of $V$. When $M$ is the whole monoid $\operatorname{Hyp}(\tau)$, an $M$-hyperidentity is called a hyperidentity, and an $M$-solid variety is called a solid variety.

Let $\mathcal{M}$ be any submonoid of $\mathcal{H} y p(\tau)$. Since $M$ contains the identity hypersubstitution, any $M$-hyperidentity of a variety $V$ is an identity of $V$. This means that the relation of $M$-hypersatisfaction, defined between $\operatorname{Alg}(\tau)$ and $W_{\tau}(X)^{2}$, is a subrelation of the relation of satisfaction from which we induced our Galois-connection (Id, Mod). The new Galois-connection induced by the relation of $M$-hypersatisfaction is called $\left(H_{M}\right.$ Mod, $\left.H_{M} I d\right)$ and is defined on classes $K$ and sets $\Sigma$ as follows:

$$
\begin{gathered}
H_{M} I d K= \\
\left\{s \approx t \in W_{\tau}(X)^{2} \mid s \approx t \text { is an } M-\text { hyperidentity of } \mathcal{A} \text { for all } \mathcal{A} \in K\right\}, \\
H_{M} M \text { od } \Sigma= \\
\{\mathcal{A} \in \operatorname{Alg}(\tau) \mid \text { all identities in } \Sigma \text { are } M-\text { hyperidentities of } \mathcal{A}\} .
\end{gathered}
$$

The Galois-closed classes of algebras under this connection are the $M$ solid varieties of type $\tau$, which form a complete sublattice of the lattice of all varieties of type $\tau$. Thus studying $M$-solid and solid varieties is a way to study complete sublattices of the lattice of all varieties of a given type.

We now introduce some closure operators on the two sets $A l g(\tau)$ and $W_{\tau}(X)^{2}$. For equations we define

$$
\chi_{M}^{E}[u \approx v]:=\{\hat{\sigma}[u] \approx \hat{\sigma}[v] \mid \sigma \in M\} .
$$

For any set $\Sigma$ of identities we set 


$$
\chi_{M}^{E}[\Sigma]=\bigcup_{u \approx v \in \Sigma} \chi_{M}^{E}[u \approx v] .
$$

Hypersubstitutions can also be applied to algebras, as follows. Given an algebra $\mathcal{A}=\left(A ;\left(f_{i}^{\mathcal{A}}\right)_{i \in I}\right)$ and a hypersubstitution $\sigma$, we define the algebra $\sigma(\mathcal{A})=\left(A ;\left(f_{i}^{\sigma(\mathcal{A})}\right)_{i \in I}\right):=\left(A ;\left(\sigma\left(f_{i}\right)^{\mathcal{A}}\right)_{i \in I}\right)$. This algebra is called the derived algebra determined by $\mathcal{A}$ and $\sigma$. Notice that by definition it is of the same type as the algebra $\mathcal{A}$. Now we define an operator $\chi_{M}^{A}$ on the set $A l g(\tau)$, first on individual algebras and then on classes $K$ of algebras, by

$$
\chi_{M}^{A}[\mathcal{A}]=\{\sigma[\mathcal{A}] \mid \sigma \in M\}
$$

and

$$
\chi_{M}^{A}[K]=\bigcup_{\mathcal{A} \in K} \chi_{M}^{A}[\mathcal{A}]
$$

If $M=\operatorname{Hyp}(\tau)$ the operators are denoted by $\chi^{A}$ and $\chi^{E}$.

Let $\tau$ be a fixed type and let $\mathcal{M}$ be any submonoid of $\mathcal{H} y p(\tau)$. The two operators $\chi_{M}^{E}$ and $\chi_{M}^{A}$ are closure operators and are connected by the condition

$$
\chi_{M}^{A}[\mathcal{A}] \text { satisfies } u \approx v \text { iff } \mathcal{A} \text { satisfies } \chi_{M}^{E}[u \approx v] .
$$

The following propositions are also obvious (see [1] or [2]).

Theorem 1.2. Let $K \subseteq A l g(\tau)$ and $\Sigma \subseteq W_{\tau}(X)$. Then there holds

$$
\begin{array}{lll}
\text { (i) } & H_{M} \operatorname{Mod} \Sigma & =\operatorname{Mod} \chi_{M}^{E}[\Sigma], \\
\text { (ii) } & H_{M} \operatorname{Mod} \Sigma & \subseteq \operatorname{Mod} \Sigma, \\
\text { (iii) } & \chi_{M}^{A}[\operatorname{H} M o d \Sigma] & =H_{M} \operatorname{Mod} \Sigma, \\
\text { (iv) } & \chi_{M}^{E}\left[\operatorname{IdH} H_{M} \operatorname{Mod} \Sigma\right] & =\operatorname{Id} H_{M} \operatorname{Mod} \Sigma, \\
\text { (v) } & H_{M} \operatorname{Mod} H_{M} I d K & =\operatorname{Mod} I d \chi_{M}^{A}[K], \\
\text { (i') } & H_{M} I d K & =\operatorname{Id} \chi_{M}^{A}[K], \\
\text { (ii') } & H_{M} I d K & \subseteq \operatorname{IdK}, \\
\text { (iii') } & \chi_{M}^{E}\left[H_{M} I d K\right] & =H_{M} I d K, \\
\text { (iv') } & \chi_{M}^{A}\left[\operatorname{Mod} H_{M} I d K\right] & =\operatorname{Mod} H_{M} I d K, \\
\text { (v') } & H_{M} I d H_{M} \operatorname{Mod} \Sigma & =\operatorname{Id} \operatorname{Mod} \chi_{M}^{E}[\Sigma] .
\end{array}
$$

$M$-solid varieties can be characterized by the following theorem:

Theorem 1.3. Let $\mathcal{M}$ be a monoid of hypersubstitutions of type $\tau$. For any variety $V$ of type $\tau$, the following conditions are equivalent: 
(i) $V=H_{M} M o d H_{M} I d V$

(ii) $\chi_{M}^{A}[V]=V$,

(iii) $I d V=H_{M} I d V$,

(iv) $\chi_{M}^{E}[I d V]=I d V$.

And dually, for any equational theory $\Sigma$ of type $\tau$, the following conditions are equivalent:

$$
\begin{aligned}
& \text { (i') } \Sigma \quad=H_{M} I d H_{M} \operatorname{Mod} \Sigma, \\
& \text { (ii') } \chi_{M}^{E}[\Sigma] \quad=\Sigma \text {, } \\
& \text { (iii') } \operatorname{Mod} \Sigma=H_{M} \operatorname{Mod} \Sigma \text {, } \\
& \text { (iv') } \chi_{M}^{A}[\operatorname{Mod} \Sigma]=\operatorname{Mod} \Sigma \text {. }
\end{aligned}
$$

A variety which satisfies the condition (i) is called an $M$ - hyperequational class.

The subrelation $\underset{M-h y p}{\models}$ of $\models$ satisfies the following condition:

$\forall K \subseteq \operatorname{Alg}(\tau) \forall \Sigma \subseteq W_{\tau}(X)^{2}\left(\left(H_{M} \operatorname{Mod} \Sigma=K \wedge H_{M} I d K=\Sigma\right) \Rightarrow\right.$ $\operatorname{Mod} \Sigma=K \wedge I d \bar{K}=\Sigma)$.

Such subrelations are called Galois-closed. The Galois connections induced by Galois-closed subrelations of a given relation generate complete sublattices of the complete lattices generated by the Galois connection induced by that given relation [3]. Moreover we have the following result.

Theorem 1.4. Let $\mathcal{M}$ be a monoid of hypersubstitutions of type $\tau$. Then the class $\mathcal{S}_{M}(\tau)$ of all $M$-solid varieties of type $\tau$ forms a complete sublattice of the lattice $\mathcal{L}(\tau)$ of all varieties of type $\tau$. Dually, the class of all $M$-hyperequational theories forms a complete sublattice of the lattice of all equational theories of type $\tau$.

When $\mathcal{M}_{1}$ and $\mathcal{M}_{2}$ are both submonoids of $\mathcal{H} y p(\tau)$ and $\mathcal{M}_{1}$ is a submonoid of $\mathcal{M}_{2}$, then the corresponding complete lattices satisfy $\mathcal{S}_{M_{2}}(\tau)$ $\subseteq \mathcal{S}_{M_{1}}(\tau)$. As a special case, for any $\mathcal{M} \subseteq \mathcal{H} y p(\tau)$ we see that the lattice $\mathcal{S}(\tau)$ of all solid varieties of type $\tau$ is always a sublattice of the lattice $\mathcal{S}_{M}(\tau)$. At the other extreme, for the smallest possible submonoid $\mathcal{M}=\left\{\sigma_{i d}\right\}$ the corresponding lattice of $M$-solid varieties is the whole lattice $\mathcal{L}(\tau)$ of all varieties of type $\tau$. Thus we obtain a range of complete sublattices of $\mathcal{L}(\tau)$ to $\mathcal{S}(\tau)$.

Our aim is to transfer this theory to another kind of hypersubstitution and to colored terms. 


\section{Multi-Hypersubstitutions And COLORATIOns OF TERMS}

In a term a certain operation symbol may occur more than once. To distinguish between the different occurrences of the same operation symbol we assign to each occurrence of any operation symbol a color. Representing a term by a tree, we get a vertex-colored graph. To apply different hypersubstitutions to the same operation symbol, if it is differently colored, we define the concept of a multi-hypersubstitution.

Definition 2.1. A map $\rho$ from $\mathbb{N}$ into $H y p(\tau)$ is called a multi- hypersubstitution. Let $H y p^{\mathbb{N}}$ be the set of all multi-hypersubstitutions.

To distinguish between different occurrences of the same operation symbol in a term $t$ we assign to each operation symbol in $t$ an address in $t$, i.e. an element of a given set. Usually adresses are sequences of natural numbers. Let $a d d(t)$ be the set of all addresses of the term $t$. We introduce the concept of a coloration to allow that equal operation symbols at different places are considered differently. On the other hand, a coloration allows that equal operation symbols with different addresses are equally colored. A coloration of a type $\tau$ is defined in the following way.

Definition 2.2. Any mapping $\alpha_{t}$ from $\operatorname{add}(t), t \in W_{\tau}(X) \backslash X$, into $\mathbb{N}$ is called a coloration of the term $t$. We denote by $C(t)$ the set of all colorations of the term $t$. A set $C \subseteq \bigcup\left\{C(r) \mid r \in W_{\tau}(X)\right\}$ with $|C(r) \cap C|=1$ for all $r \in W_{\tau}(X) \backslash X$ is called a coloration of $W_{\tau}(X)$.

Using colorations, multi- hypersubstitutions can be extended to mappings defined on terms. In a first step we extend multi- hypersubstitutions to mappings from the set $S u b(t)$ of all subterms of a given term $t$ to the set of terms.

Definition 2.3. Let $C$ be a coloration of $W_{\tau}(X), t \in W_{\tau}(X)$ with the coloration $\alpha \in C, s \in S u b(t)$, and let $\rho$ be a multi-hypersubstitution.

(i) If $s \in X$ then $\widehat{\rho}_{C, t}[s]:=s$.

(ii) If $s=f_{i}\left(s_{1}, \ldots, s_{n_{i}}\right)$ with $i \in I$ and $s_{1}, \ldots, s_{n_{i}} \in S u b(t)$, where $f_{i}$ has the address $a$ in $t$ then

$$
\widehat{\rho}_{C, t}[s]:=\rho(\alpha(a))\left(f_{i}\right)\left(\widehat{\rho}_{C, t}\left[s_{1}\right], \ldots, \widehat{\rho}_{C, t}\left[s_{n_{i}}\right]\right)
$$

Using the mappings $\widehat{\rho}_{C, t}[t]: S u b(t) \rightarrow W_{\tau}(X)$ for terms $t \in W_{\tau}(X)$ we can extend multi-hypersubstitutions to mappings defined on terms. 
Definition 2.4. Let $C$ be a coloration of $W_{\tau}(X)$ and $\alpha \in C$ be the coloration of a term $t \in W_{\tau}(X)$. Then for a multi-hypersubstitution $\rho$ we put $\widehat{\rho}_{C}[t]:=\widehat{\rho}_{C, t}[t]$.

The following example shows that the composition of two multi- hypersubstitutions does not be a multi- hypersubstitution. For this we consider the type $\tau=(2)$, the terms $s=f(y, f(y, x))$ and $t=f(f(x, y), y)$ withe the following coloration $C$ :

$\alpha_{t}(a)=0$ for all $a \in \operatorname{add}(t)$;

$\alpha_{s}(q)=0$, where $s$ is the address of the leftmost $f$ in $s$;

$\alpha_{s}(a)=1$ for all $a \in \operatorname{add}(s), a \neq q$.

Let $\rho \in \operatorname{Hyp}(\tau)^{\mathbb{N}}$ be a multi-hypersubstitution with $\rho(0)=\sigma_{y x}$ and $\rho(a)=\sigma_{i d}$ for $a \in \mathbb{N} \backslash\{0\}$. Then we have $\widehat{\rho}_{C}[t]=s$ and $\widehat{\rho}_{C}[t]=$ $f(f(y, x), y)$. Since all operation symbols in $t$ have the same color, we can not find a multi-hypersubstitution which provides $f(f(y, x), y)$ by application on $t$.

If all addresses of a term have the same color, then a multi- hypersubstitution can be replaced on that term by one of its components, i.e. by an ordinary hypersubstitution as the following lemma shows.

Lemma 2.5. Let $C$ be a coloration of $W_{\tau}(X), \rho \in H y p(\tau)^{\mathbb{N}}$, and $t \in$ $W_{\tau}(X)$ such that there is an $n \in \mathbb{N}$ with $\alpha_{t}(a)=n$ for all $a \in \operatorname{add}(t)$. Then $\widehat{\rho}_{C}[t]=\widehat{\rho(n)}[t]$.

Proof: Since $\widehat{\rho}_{C}[t]=\widehat{\rho}_{C, t}[t]$ we show by induction that $\widehat{\rho(n)}[s]:=\widehat{\rho}_{C, t}[s]$ for each $s \in S u b(t)$. If $s \in X$ then $\widehat{\rho}_{C, t}[s]=s=\widehat{\rho(n)}[s]$.

Let $s=f_{i}\left(s_{1}, \ldots, s_{n_{i}}\right)$ and suppose that $\widehat{\rho}_{C, t}\left[s_{j}\right]=\widehat{\rho(n)}\left[s_{j}\right]$ for $1 \leq j \leq$ $n_{i}$ then

$$
\begin{aligned}
& \widehat{\rho}_{C, t}\left[f_{i}\left(s_{1}, \ldots, s_{n_{i}}\right)\right] \\
&= \rho\left(\alpha_{t}(a)\right)\left(f_{i}\right)\left(\widehat{\rho}_{C, t}\left[s_{1}\right], \ldots, \widehat{\rho}_{C, t}\left[s_{n_{i}}\right]\right) \\
&\left(a \text { denotes the address of } f_{i} \text { in } t\right) \\
&= \rho\left(\alpha_{t}(a)\right)\left(f_{i}\right)\left(\widehat{\rho(n)}\left[s_{1}\right], \ldots, \widehat{\rho(n)}\left[s_{n_{i}}\right]\right) \\
&(\text { by the hypothesis }) \\
&= \rho(n)\left(f_{i}\right)\left(\widehat{\rho(n)}\left[s_{1}\right], \ldots, \widehat{\rho(n)}\left[s_{n_{i}}\right]\right) \\
&= \widehat{\rho(n)}\left[f_{i}\left(s_{1}, \ldots, s_{n_{i}}\right)\right] .
\end{aligned}
$$




\section{Colored solid VArieties}

Using multi-hypersubstitutions we define operators corresponding to $\chi_{M}^{A}, \chi_{M}^{E}$ of the introduction and apply these operators to sets of equations and to classes of algebras.

Definition 3.1. Let $C$ be a coloration of $W_{\tau}(X)$ and $\Sigma \subseteq W_{\tau}(X)^{2}$. Then we put $\chi_{C}^{e}[\Sigma]:=\left\{\widehat{\rho}_{C}[u] \approx \widehat{\rho}_{C}[v] \mid u \approx v \in \Sigma, \rho \in \operatorname{Hyp}(\tau)^{\mathbb{N}}\right\}=$ $\chi_{C}^{e, 0}[\Sigma]$. For $n \in \mathbb{N}$, we put $\chi_{C}^{e, n+1}[\Sigma]:=\chi_{C}^{e,}\left[\chi_{C}^{e, n}[\Sigma]\right]$. Let $\chi_{C}^{E}[\Sigma]:=$ $\bigcup\left\{\chi_{C}^{e, n}[\Sigma] \mid n \in \mathbb{N}\right\}$.

For any set $\Sigma \subseteq W_{\tau}(X)^{2}$ we have $\chi_{C}^{e}[\Sigma]=\Sigma$ iff $\chi_{C}^{E}[\Sigma]=\Sigma$. Indeed, If $\chi_{C}^{e}[\Sigma]=\Sigma$ then it is easy to see that $\chi_{C}^{e, n}[\Sigma]=\Sigma$ for all $n \in \mathbb{N}$ and thus $\chi_{C}^{E}[\Sigma]=\Sigma$. Conversely, if $\chi_{C}^{E}[\Sigma]=\Sigma$ then $\chi_{C}^{e}[\Sigma] \subseteq \chi_{C}^{E}[\Sigma]$ provides $\chi_{C}^{e}[\Sigma] \subseteq \Sigma$. Since $\rho_{i d} \in \operatorname{Hyp}(\tau)^{\mathbb{N}}$ we have $\Sigma \subseteq \chi_{C}^{e}[\Sigma]$ and altogether, $\chi_{C}^{e}[\Sigma]=\Sigma$.

Definition 3.2. Let $C$ be a coloration of $W_{\tau}(X)$ and let $V$ be a variety of type $\tau$. $V$ is called $C$-colored solid if $I d V=\chi_{C}^{E}[I d V]$.

Example 3.3. We consider the following example of a $C$-colored variety of type $\tau=(2)$. Let $R B=\operatorname{Mod}\left\{x(y z) \approx(x y) z \approx x z, x^{2} \approx x\right\}$ be the variety of rectangular bands. It is well-known that $R B$ is solid (see e.g. [6]). The set $I d R B$ of all identities satisfied in $R B$ is the set of all equations $s \approx t$ such that the first variable of $s$ agrees with the first variable of $t$ and the last variable of $s$ agrees with the last variable of $t$. For $t \in W_{(2)}(X)$ such that $t$ starts and ends with the same variable we define $\alpha_{t}: \operatorname{add}(t) \rightarrow \mathbb{N}$ with $\alpha_{t}(a)=1$ for all $a \in \operatorname{add}(t)$ and if $t$ starts and ends with different variables, then we define $\alpha_{t}(a)=2$ for all $a \in \operatorname{add}(t)$. Let $s \approx t \in I d R B$ and let $\rho$ be a multi-hypersubstitution. Then it is easy to see that $\widehat{\rho_{C}}[s] \approx \widehat{\rho_{C}}[t] \in \operatorname{IdRB}$ using solidity.

Definition 3.4. Let $C$ be a coloration of $W_{\tau}(X)$, let $\rho \in \operatorname{Hyp}(\tau)^{\mathbb{N}}$ and let $\mathcal{A}=\left(A ;\left(f_{i}^{\mathcal{A}}\right)_{i \in I}\right)$ be an algebra of type $\tau$. Then we define:

$\rho[\mathcal{A}]:=\left(A ;\left(f_{i}^{\rho[\mathcal{A}]}\right)_{i \in I}\right)$ where $f_{i}^{\rho[\mathcal{A}]}=\widehat{\rho}_{C}\left[f_{i}\left(x_{1}, \ldots, x_{n_{i}}\right)\right]^{\mathcal{A}}$ for $i \in I$.

Definition 3.5. Let $C$ be a coloration of $W_{\tau}(X)$ and $K$ be a class of algebras of type $\tau$. Then we put $\chi_{C}^{A}[K]:=\{\rho[\mathcal{A}] \mid \mathcal{A} \in K, \rho \in$ $\left.\operatorname{Hyp}(\tau)^{\mathbb{N}}\right\}$.

It is easy to check that $\chi_{C}^{E}$ and $\chi_{C}^{A}$ have the properties of a completely additive closure operator. From Lemma 3.6 below will follow that a $C$ colored solid variety is solid. Our new closure operators are connected with the operators defined in the Introduction.

Lemma 3.6. Let $C$ be a coloration of $W_{\tau}(X)$. Then 
(i) $\chi^{E}[\Sigma] \subseteq \chi_{C}^{E}[\Sigma]$ for each $\Sigma \subseteq W_{\tau}(X)^{2}$.

(ii) $\chi_{C}^{A}[K]=\chi^{A}[K]$ for each $K \subseteq \operatorname{Alg}(\tau)$.

Proof: (i) $\chi_{C}^{e}[\Sigma] \subseteq \chi_{C}^{E}[\Sigma]$ we have to show that $\chi^{E}[\Sigma] \subseteq \chi_{C}^{e}[\Sigma]$. Let $\sigma \in \operatorname{Hyp}(\tau)$. Then we consider the multi-hypersubstitution $\rho \in$ $\operatorname{Hyp}(\tau)^{\mathbb{N}}$ with $\rho(a)=\sigma$ for all $a \in \mathbb{N}$. If $t \in W_{\tau}(X)$ then we will check that $\widehat{\sigma}[t]=\widehat{\rho}_{C}[t]$. Since $\widehat{\rho}_{C}[t]=\widehat{\rho}_{C, t}[t]$ we show that $\widehat{\sigma}[s]:=\widehat{\rho}_{C, t}[s]$ for each $s \in S u b(t)$ by induction on the complexity of the term $s$.

If $s \in X$ then $\widehat{\sigma}[s]=s=\widehat{\rho}_{C, t}[s]$.

Assume that $s=f_{i}\left(s_{1}, \ldots, s_{n_{i}}\right)$ with $i \in I$ and $s_{1}, \ldots, s_{n_{i}} \in S u b(t)$ and suppose inductively that $\widehat{\sigma}\left[s_{k}\right]=\widehat{\rho}_{C, t}\left[s_{k}\right]$ for $1 \leq k \leq n_{i}$. Then for a suitable $a \in \mathbb{N}$ we have

$$
\begin{gathered}
\widehat{\rho}_{C, t}[s]=\rho(a)\left(f_{i}\right)\left(\widehat{\rho}_{C, t}\left[s_{1}\right], \ldots, \widehat{\rho}_{C, t}\left[s_{n_{i}}\right]\right) \\
=\sigma\left(f_{i}\right)\left(\widehat{\sigma}\left[s_{1}\right], \ldots, \widehat{\sigma}\left[s_{n_{i}}\right]\right)=\widehat{\sigma}\left[f_{i}\left(s_{1}, \ldots, s_{n_{i}}\right)\right] .
\end{gathered}
$$

(ii) Let $\mathcal{A} \in K$. For $i \in I$ let $a_{i}$ be the color of $f_{i}$ in the fundamental term $f_{i}\left(x_{1}, \ldots, x_{n_{i}}\right)$.

Let $\rho \in \operatorname{Hyp}(\tau)^{\mathbb{N}}$. Then we consider the hypersubstitution $\sigma \in$ $\operatorname{Hyp}(\tau)$ with $\sigma\left(f_{i}\right)=\rho\left(a_{i}\right)\left(f_{i}\right)$ for $i \in I$ and have

$$
\begin{gathered}
f_{i}^{\rho[\mathcal{A}]}=\widehat{\rho}_{C}\left[f_{i}\left(x_{1}, \ldots, x_{n_{i}}\right)\right]^{\mathcal{A}}=\left(\rho\left(a_{i}\right)\left(f_{i}\right)\left(x_{1}, \ldots, x_{n_{i}}\right)\right)^{\mathcal{A}} \\
=\sigma\left(f_{i}\right)\left(x_{1}, \ldots, x_{n_{i}}\right)^{\mathcal{A}}=\sigma\left(f_{i}\right)^{\mathcal{A}} .
\end{gathered}
$$

This shows that $\chi_{C}^{A}[\mathcal{A}] \subseteq \chi^{A}[\mathcal{A}]$.

Let $\sigma \in \operatorname{Hyp}(\tau)$. Then we consider the multi-hypersubstitution $\rho \in$ $\operatorname{Hyp}(\tau)^{\mathbb{N}}$ with $\rho(a)=\sigma$ for all $a \in \mathbb{N}$. Now we have

$$
\begin{aligned}
f_{i}^{\sigma[\mathcal{A}]}=\sigma\left(f_{i}\right)^{\mathcal{A}}= & \left(\sigma\left(f_{i}\right)\left(x_{1}, \ldots, x_{n_{i}}\right)\right)^{\mathcal{A}}=\left(\rho\left(a_{i}\right)\left(f_{i}\right)\left(x_{1}, \ldots, x_{n_{i}}\right)\right)^{\mathcal{A}} \\
& =\widehat{\rho}_{C}\left[f_{i}\left(x_{1}, \ldots, x_{n_{i}}\right)\right]^{\mathcal{A}}=f_{i}^{\rho[\mathcal{A}]}
\end{aligned}
$$

This shows that $\chi^{A}[\mathcal{A}] \subseteq \chi_{C}^{A}[\mathcal{A}]$. Altogether we have $\chi_{C}^{A}[\mathcal{A}]=\chi^{A}[\mathcal{A}]$ and thus $\chi_{C}^{A}[K]=\chi^{A}[K]$.

Using the operators $\chi_{C}^{A}$ and $\chi_{C}^{E}$ we define two new relations between $\operatorname{Alg}(\tau)$ and $W_{\tau}(X)^{2}$.

Definition 3.7. Let $C$ be a coloration of $W_{\tau}(X)$. Then we put

$$
\begin{aligned}
& R_{1}:=\left\{(\mathcal{A}, s \approx t) \mid \mathcal{A} \in \operatorname{Alg}(\tau), s \approx t \in W_{\tau}(X)^{2}, \chi_{C}^{E}[s \approx t] \subseteq I d \mathcal{A}\right\} \\
& R_{2}:=\left\{(\mathcal{A}, s \approx t) \mid \mathcal{A} \in \operatorname{Alg}(\tau), s \approx t \in W_{\tau}(X)^{2}, s \approx t \in I d \chi_{C}^{A}[\mathcal{A}]\right\} \\
& \mathcal{C} M o d \Sigma:=\left\{\mathcal{A} \mid\{\mathcal{A}\} \times \Sigma \subseteq R_{1}\right\} \text { for } \Sigma \subseteq W_{\tau}(X)^{2} ;
\end{aligned}
$$


$\mathcal{C} I d K:=\left\{s \approx t \mid K \times\{s \approx t\} \subseteq R_{1}\right\}$ for $K \subseteq \operatorname{Alg}(\tau)$.

Because of Lemma 3.6 (ii) the relation $R_{2}$ agrees with the relation $\underset{\text { hyp }}{\models}$. Since $\underset{h y p}{\models}$ is a Galois closed subrelation of $\models$, the relation $R_{2}$ has the same property. Since we are more interested in $R_{1}$, we ask whether $R_{1}$ is a Galois closed subrelation of $\models$. At first we have the following property:

Proposition 3.8. Let $C$ be a coloration of $W_{\tau}(X)$. Then $R_{1}$ is a subrelation of $\models$ such that for $K \subseteq A \lg (\tau)$ and all $\Sigma \subseteq W_{\tau}(X)^{2}$ the following holds: If $\Sigma=\mathcal{C} I d K$ and $K=\mathcal{C} M o d \Sigma$ then $K=\operatorname{Mod} \Sigma$.

Proof: At first we show that $R_{1}$ is a subrelation of $\models$. For this let $(\mathcal{A}, s \approx t) \in R_{1}$. Then $\chi_{C}^{E}[s \approx t] \subseteq I d \mathcal{A}$ and $s \approx t \in I d \mathcal{A}$ since $\chi_{C}^{E}$ is a closure operator. Thus $(\mathcal{A}, s \approx t) \in \models$.

Now we show that $\Sigma=\chi_{C}^{E}[\Sigma]$. Since $\chi_{C}^{E}$ is a closure operator, we have $\Sigma \subseteq \chi_{C}^{E}[\Sigma]$.

Conversely let $u \approx v \in \chi_{C}^{E}[\Sigma]$. Then there is an $s \approx t \in \Sigma$ with $u \approx v \in \chi_{C}^{E}[s \approx t]$, i.e. $\chi_{C}^{E}[u \approx v] \subseteq \chi_{C}^{E}\left[\chi_{C}^{E}[s \approx t]\right] \subseteq \chi_{C}^{E}[s \approx t]$. From $s \approx t \in \Sigma=\mathcal{C} I d K$ it follows $\chi_{C}^{E}[s \approx t] \subseteq I d K$ and $\chi_{C}^{E}[u \approx v] \subseteq \chi_{C}^{E}[s \approx$ $t] \subseteq I d K$, i.e. $u \approx v \in \mathcal{C} I d K$. This shows $\chi_{C}^{E}[\Sigma] \subseteq \Sigma$. Now we have

$$
\begin{aligned}
\operatorname{Mod} \Sigma & =\{\mathcal{A} \mid \mathcal{A} \in \operatorname{Alg}(\tau), \Sigma \subseteq I d \mathcal{A}\} \\
& =\left\{\mathcal{A} \mid \mathcal{A} \in \operatorname{Alg}(\tau), \chi_{C}^{E}[\Sigma] \subseteq I d \mathcal{A}\right\} \\
& =\mathcal{C} \operatorname{Mod} \Sigma \\
& =K .
\end{aligned}
$$

To obtain a characterization of colored solid varieties we check at first the conditions of Theorem 1.2 for M-solid varieties.

Lemma 3.9. Let $C$ be a coloration of $W_{\tau}(X)$, let $\Sigma \subseteq W_{\tau}(X)^{2}$, and let $K$ be a class of algebras of type $\tau$. Then

$$
\chi_{C}^{E}[\Sigma] \subseteq I d K \Leftrightarrow \chi_{C}^{E}[\Sigma] \subseteq I d \chi_{C}^{A}[K]
$$

Proof: Because of $K \subseteq \chi_{C}^{A}[K] \Rightarrow I d \chi_{C}^{A}[K] \subseteq I d K$ we get $\chi_{C}^{E}[\Sigma] \subseteq$ $I d \chi_{C}^{A}[K] \Rightarrow \chi_{C}^{E}[\Sigma] \subseteq I d K$.

By Lemma $3.6(\mathrm{i})$ and the definition of the closure operators $\chi_{C}^{E}$ and $\chi^{E}$ we have $\chi_{C}^{E}[\Sigma]=\chi_{C}^{E}\left[\chi_{C}^{E}[\Sigma]\right] \supseteq \chi^{E}\left[\chi_{C}^{E}[\Sigma]\right] \supseteq \chi_{C}^{E}[\Sigma]$, i.e. $\chi_{C}^{E}[\Sigma]=$ $\chi^{E}\left[\chi_{C}^{E}[\Sigma]\right]$ 
Consequently we have

$$
\chi_{C}^{E}[\Sigma] \subseteq I d K \Rightarrow \chi^{E}\left[\chi_{C}^{E}[\Sigma]\right] \subseteq I d K \Rightarrow \chi_{C}^{E}[\Sigma] \subseteq I d \chi^{A}[K],
$$

since $\left(\chi^{E}, \chi^{A}\right)$ is a conjugate pair. Then by Lemma [3.6, $\chi_{C}^{E}[\Sigma] \subseteq$ $\operatorname{Id} \chi_{C}^{A}[K]$.

As a consequence we have

$$
\mathcal{C} M o d \Sigma=\left\{\mathcal{A} \mid \mathcal{A} \in \operatorname{Alg}(\tau) \text { and } \chi_{C}^{E}[\Sigma] \subseteq I d \chi_{C}^{A}[\mathcal{A}]\right\} .
$$

Now we prove that the sets of the form $\mathcal{C} I d K$ and $\mathcal{C} M o d \Sigma$ are closed under the operators $\chi_{C}^{E}$ and $\chi_{C}^{A}$, respectively.

Lemma 3.10. Let $C$ be a coloration of $W_{\tau}(X)$.

(i) For $K \subseteq A \lg (\tau)$ there holds $\chi_{C}^{E}[\mathcal{C} I d K]=\mathcal{C} I d K$.

(ii) For $\Sigma \subseteq W_{\tau}(X)^{2}$ there holds $\chi_{C}^{A}[\mathcal{C} M o d \Sigma]=\mathcal{C} M o d \Sigma$.

Proof: (i) Clearly, $\mathcal{C} I d K \subseteq \chi_{C}^{E}[\mathcal{C} I d K]$. Let $u \approx v \in \chi_{C}^{E}[\mathcal{C} I d K]$. Then there is an equation $s \approx t \in \mathcal{C} I d K$ with $u \approx v \in \chi_{C}^{E}[s \approx t]$. Since $\chi_{C}^{E}$ is a closure operator we have $\chi_{C}^{E}[u \approx v] \subseteq \chi_{C}^{E}\left[\chi_{C}^{E}[s \approx t]\right]=\chi_{C}^{E}[s \approx t]$. From $s \approx t \in \mathcal{C} I d K$ it follows $\chi_{C}^{E}[s \approx t] \subseteq I d K$. Then $\chi_{C}^{E}[u \approx v] \subseteq$ $I d K$, thus $u \approx v \in \mathcal{C} I d K$.

(ii) Clearly, $\mathcal{C} \operatorname{Mod} \Sigma \subseteq \chi_{C}^{A}[\mathcal{C} \operatorname{Mod} \Sigma]$. Let $\mathcal{A} \in \chi_{C}^{A}[\mathcal{C} \operatorname{Mod} \Sigma]$. Then there is a $\mathcal{B} \in \mathcal{C} M o d \Sigma$ such that $\mathcal{A} \in \chi_{C}^{A}[\mathcal{B}]$. This implies $\chi_{C}^{A}[\mathcal{A}] \subseteq$ $\chi_{C}^{A}\left[\chi_{C}^{A}[\mathcal{B}]\right]=\chi_{C}^{A}[\mathcal{B}]$ and $\operatorname{Id} \chi_{C}^{A}[\mathcal{B}] \subseteq \operatorname{Id} \chi_{C}^{A}[\mathcal{A}]$. From $\mathcal{B} \in \mathcal{C} M o d \Sigma$ it follows $\chi_{C}^{E}[\Sigma] \subseteq I d \chi_{C}^{A}[\mathcal{B}]$ by $(*)$. Thus $\chi_{C}^{E}[\Sigma] \subseteq I d \chi_{C}^{A}[\mathcal{A}]$ and $\mathcal{A} \in \mathcal{C} \operatorname{Mod} \Sigma$ by $(*)$. This shows that $\chi_{C}^{A}[\mathcal{C} \operatorname{Mod} \Sigma] \subseteq \mathcal{C} M o d \Sigma$.

Because of $R_{1} \subseteq \models$, we have $\mathcal{C} M o d \Sigma \subseteq \operatorname{Mod} \Sigma$ and $\mathcal{C} I d K \subseteq \operatorname{IdK}$ for all sets $\Sigma \subseteq W_{\tau}(X)^{2}$ and for all $K \subseteq \overline{A l} g(\tau)$.

The following example shows that $R_{1}$ is not a Galois closed subrelation of $\models$. We consider the set of all identities of the greatest solid variety $V_{H S}:=\operatorname{Mod}\left\{x_{1}\left(x_{2} x_{3}\right) \approx\left(x_{1} x_{2}\right) x_{3}, x_{1}^{2} \approx x_{1}^{4}, x_{1} x_{2} x_{1} x_{3} x_{1} x_{2} x_{1} \approx\right.$ $\left.x_{1} x_{2} x_{3} x_{2} x_{1}, x_{1}^{2} x_{2}^{2} x_{3} \approx x_{1}^{2} x_{2} x_{1}^{2} x_{2} x_{3}, x_{1} x_{2}^{2} x_{3}^{2} \approx x_{1} x_{2} x_{3}^{2} x_{2} x_{3}^{2}\right\}$ of semigroups ([5]).

Example 3.11. Let $\tau=(2)$ and let $f$ be the binary operation symbol. Then there is a coloration $C$ of $W_{(2)}(X)$ such that $R_{1}$ is not a Galoisclosed subrelation of $\models$. We will show that there is a set $\Sigma$ of equations with

$$
\Sigma=C I d V_{H S} \text { and } V_{H S}=C M o d \Sigma \not \Sigma=I d V_{H S} .
$$

We put $s:=f(f(x, x), f(f(x, x), f(x, x)))$ and $\alpha_{s}: \operatorname{add}(s) \longrightarrow \mathbb{N}$ with $\alpha_{s}(a)=1$ for all $a \in \operatorname{add}(s)$. 
We set $\Psi:=\left(\left\{s \approx t \mid t \in W_{(2)}(X) \backslash\{s\}\right\} \cup\left\{t \approx s \mid t \in W_{(2)}(X) \backslash\right.\right.$ $\{s\}\}) \cap I d V_{H S}$ and $\Sigma:=I d V_{H S} \backslash \Psi$.

For $t \in W_{(2)}(X) \backslash\{s\}$ we define $\alpha_{t}: \operatorname{add}(t) \longrightarrow \mathbb{N}$ with $\alpha_{t}(a)=0$ for all $a \in \operatorname{add}(t)$.

This gives us a coloration $C$ of $W_{(2)}(X)$.

If $t \in W_{(2)}(X) \backslash\{s\}$ then for $\rho \in \operatorname{Hyp}(2)^{\mathbb{N}}$ holds $\widehat{\rho}[t]=\widehat{\rho(0)}[t]$ by Lemma 2.5

As a consequence we have (together with Lemma 3.6) $\chi_{C}^{e}[\Theta]=\chi^{E}[\Theta]$ for each set $\Theta \subseteq W_{(2)}(X)^{2}$. Since $\chi^{E}$ is a closure operator, we have the idempotence propertyand it is easy to check that $\chi_{C}^{e, n}[\Sigma]=\chi^{E}[\Sigma]$ for all $n \in \mathbb{N}$. This shows that $\chi_{C}^{E}[\Sigma]=\chi^{E}[\Sigma]$.

Hence $\mathcal{C} M o d \Sigma=\left\{\mathcal{A} \mid\{\mathcal{A}\} \times \Sigma \subseteq R_{1}\right\}=\left\{\mathcal{A} \mid \mathcal{A} \in \operatorname{Alg}(\tau), \chi_{C}^{E}[\Sigma] \subseteq\right.$ $I d \mathcal{A}\}=\left\{\mathcal{A} \mid \mathcal{A} \in \operatorname{Alg}(\tau), \chi^{E}[\Sigma] \subseteq I d \mathcal{A}\right\}=V_{H S}$ since $V_{H S}$ is defined by the hyperidentity $f(f(x, y), z) \approx f(x, f(y, z))$.

Further we have $\mathcal{C} I d V_{H S}=\left\{r \approx t \mid V_{H S} \times\{r \approx t\} \subseteq R_{1}\right\}$

$=\left\{r \approx t \mid r \approx t \in W_{\tau}(X)^{2}, \chi_{C}^{E}[r \approx t] \subseteq I d V_{H S}\right\}$

$=\left\{r \approx t \mid r \approx t \in I d V_{H S}, \chi_{C}^{E}[r \approx t] \subseteq I d V_{H S}\right\}$

$=\left\{r \approx t \mid r \approx t \in \Sigma, \chi_{C}^{E}[r \approx t] \subseteq I d V_{H S}\right\} \cup\{r \approx t \mid r \approx t \in$ $\left.\Psi, \chi_{C}^{E}[r \approx t] \subseteq I d V_{H S}\right\}$

$=\Sigma \cup\left\{r \approx t \mid r \approx t \in \Psi, \chi_{C}^{E}[r \approx t] \subseteq I d V_{H S}\right\}$ (since $V_{H S}$ is solid )

$=\Sigma \cup \emptyset$

since the multi-hypersubstitution $\rho \in \operatorname{Hyp}(2)^{\mathbb{N}}$ with $\rho(0)=\sigma_{x y}$ and $\rho(a)=\sigma_{x}$ for $a \in \mathbb{N} \backslash\{0\}$ provides $\widehat{\rho_{C}}[s]=x$ and $\widehat{\rho_{C}}[t]=t$ for $t \in W_{(2)}(X) \backslash\{s\}$, where $s \approx t \in I d V_{H S}$ implies $t \neq x$, i.e. $\widehat{\rho_{C}}[s] \approx$ $\widehat{\rho_{C}}[t] \notin I d V_{H S}$.

Finally, because of $f(x, x) \approx s \in I d V_{H S} \backslash \Sigma$ we have $I d V_{H S} \neq \Sigma$.

Proposition 3.12. The pair $\left(\chi_{C}^{E}, \chi_{C}^{A}\right)$ is in general not a conjugate pair of completely additive closure operators.

Proof: Assume that $\left(\chi_{C}^{E}, \chi_{C}^{A}\right)$ forms a conjugate pair of completely additive closure operators. Then for all $\mathcal{A} \in \operatorname{Alg}(\tau)$ and all $s \approx t \in$ $W_{\tau}(X)^{2}$ there holds $s \approx t \in I d \chi_{C}^{A}[\mathcal{A}]$ iff $\chi_{C}^{E}[s \approx t] \subseteq I d \mathcal{A}$. In particular, $R_{1}=R_{2}$. But $R_{2}$ is a Galois-closed subrelation of $\models$ and we showed in Example 3.11 that $R_{1}$ is not Galois-closed. Thus $R_{1}$ and $R_{2}$ are different, a contradiction to $R_{1}=R_{2}$.

Since the proof of the four equivalent characterizations of $M$-solid varieties uses this property we cannot expect to have the same situation. 
Proposition 3.13. Let $C$ be a coloration of $W_{\tau}(X), K \subseteq A l g(\tau)$ and $\Sigma \subseteq W_{\tau}(X)^{2}$. Then there holds

(i) $\mathcal{C} M o d \Sigma=\operatorname{Mod} \chi_{C}^{E}[\Sigma]$,

(ii) $\mathcal{C} I d K \subseteq I d \chi_{C}^{A}[K]$, but the converse inclusion is in general not true.

Proof: (i) There holds

$$
\mathcal{A} \in \mathcal{C} M o d \Sigma \quad \Longleftrightarrow \quad \chi_{C}^{E}[\Sigma] \subseteq I d \mathcal{A} \quad \Longleftrightarrow \quad \mathcal{A} \in \operatorname{Mod} \chi_{C}^{E}[\Sigma] .
$$

(ii) Let $u \approx v \in \mathcal{C} I d K$. Then $\chi_{C}^{E}[u \approx v] \subseteq I d K$ and $\chi_{C}^{E}[u \approx v] \subseteq$ $I d \chi_{C}^{A}[K]$ by Lemma [3.9, Now we have $u \approx v \in \chi_{C}^{E}[u \approx v] \subseteq I d \chi_{C}^{A}[K]$. Altogether this shows that $\mathcal{C} I d K \subseteq I d \chi_{C}^{A}[K]$.

In order to show that the converse direction is in general not true we consider the type (2) and the coloration $C$ of $W_{(2)}(X)$ from Example 3.11. Moreover let $\Sigma \subseteq W_{\tau}(X)^{2}$ be as given in Example 3.11, Then we have $\mathcal{C} I d V_{H S}=\Sigma \neq I d V_{H S}=I d \chi^{A}\left[V_{H S}\right]=I d \chi_{C}^{A}\left[V_{H S}\right]$, since we have $V_{H S}=\chi^{A}\left[V_{H S}\right]\left(V_{H S}\right.$ is solid) and $\chi^{A}\left[V_{H S}\right]=\chi_{C}^{A}\left[V_{H S}\right]$ (Lemma 3.6).

Proposition 3.14. Let $C$ be a coloration of $W_{\tau}(X), K \subseteq A l g(\tau)$ and $\Sigma \subseteq W_{\tau}(X)^{2}$. Then there holds:

(i) $\chi_{C}^{E}[\operatorname{IdCMod} \Sigma] \supseteq \operatorname{IdC} \operatorname{Mod} \Sigma$, but the converse inclusion is in general not true,

(ii) $\chi_{C}^{A}[\operatorname{ModC} I d K]=\operatorname{ModC} I d K$.

Proof: (i) The inclusion is clear, since $\chi_{C}^{E}$ is a closure operator.

In order to show that the converse direction is in general not true we consider the type (2) and the coloration $C$ of $W_{(2)}(X)$ from Example 3.11. Moreover let $\Sigma \subseteq W_{\tau}(X)^{2}$ and $s \in W_{(2)}(X)$ be as given in Example 3.11 and $\rho \in H y p(\tau)^{\mathbb{N}}$ be defined by $\rho(0)=\sigma_{x y}$ and $\rho(a)=\sigma_{x}$ for $a \in \mathbb{N} \backslash\{0\}$. Then we get $s \approx f(x, x) \in I d V_{H S}$ and thus $\widehat{\rho}_{C}[s] \approx \widehat{\rho}_{C}[f(x, x)] \in \chi_{C}^{E}\left[\operatorname{Id} V_{H S}\right]$, i.e. $x \approx x^{2} \in \chi_{C}^{E}\left[\operatorname{Id} V_{H S}\right]$. Because of $x \approx x^{2} \notin I d V_{H S}$ we obtain $\chi_{C}^{E}\left[I d V_{H S}\right] \neq I d V_{H S}$, i.e. $\chi_{C}^{E}[\operatorname{IdC} \operatorname{Mod} \Sigma] \neq \operatorname{IdC} \operatorname{Mod} \Sigma$ since $V_{H S}=\mathcal{C} M o d \Sigma$.

(ii) Clearly, $\operatorname{Mod} \mathcal{C} I d K \subseteq \chi_{C}^{A}[\operatorname{Mod} \mathcal{C} I d K]$.

For the converse inclusion let $\mathcal{A} \in \chi_{C}^{A}[\operatorname{ModC} I d K]$. Then there is a $\mathcal{B} \in \operatorname{Mod} \mathcal{C} I d K$ with $\mathcal{A} \in \chi_{C}^{A}[\mathcal{B}]$. We want to show that $\mathcal{C} I d K \subseteq I d \mathcal{A}$. For this let $u \approx v \in \mathcal{C} I d K$, i.e. $\chi_{C}^{E}[u \approx v] \subseteq I d K$. Since $\chi_{C}^{E}$ is a closure operator we have $\chi_{C}^{E}\left[\chi_{C}^{E}[u \approx v]\right] \subseteq I d K$ and thus $\chi_{C}^{E}[u \approx v] \subseteq \mathcal{C} I d K$. Since $\mathcal{B} \in \operatorname{ModC} I d K$ we have then $\chi_{C}^{E}[u \approx v] \subseteq I d \mathcal{B}$. Lemma 3.9 shows that then $\chi_{C}^{E}[u \approx v] \subseteq I d \chi_{C}^{A}[\mathcal{B}]$. Moreover $\mathcal{A} \in \chi_{C}^{A}[\mathcal{B}]$ implies $I d \chi_{C}^{A}[\mathcal{B}] \subseteq I d \mathcal{A}$. Altogether we have $u \approx v \in \chi_{C}^{E}[u \approx v] \subseteq I d \chi_{C}^{A}[\mathcal{B}] \subseteq$ $I d \mathcal{A}$. Consequently, $\mathcal{C} I d K \subseteq I d \mathcal{A}$. 
Finally, $\mathcal{C} I d K \subseteq I d \mathcal{A}$ means $\mathcal{A} \in \operatorname{Mod} \mathcal{C} I d K$. Altogether this shows the converse inclusion $\chi_{C}^{A}[M o d \mathcal{C} I d K] \subseteq \operatorname{Mod} \mathcal{C} I d K$.

Proposition 3.15. Let $C$ be a coloration of $W_{\tau}(X), K \subseteq A \lg (\tau)$ and $\Sigma \subseteq W_{\tau}(X)^{2}$. Then there holds:

(i) $\operatorname{ModId} \chi_{C}^{A}[K] \subseteq \mathcal{C} M o d C I d K$, but the converse inclusion is in general not true;

(ii) $\mathcal{C} I d \mathcal{C} \operatorname{Mod} \Sigma \subseteq \operatorname{IdMod} \chi_{C}^{E}[\Sigma]$, but the converse inclusion is in general not true.

Proof: (i) We have

$$
\begin{aligned}
\operatorname{Mod} I d \chi_{C}^{A}[K] & \subseteq \operatorname{Mod} I d K & \text { by Proposition } 3.13 \\
& =\operatorname{Mod} \chi_{C}^{E}[\mathcal{C} I d K] & \text { by Lemma } 3.10(\mathrm{i}) \\
& =\mathcal{C} \operatorname{Mod} I d K & \text { by Proposition } 3.13(\mathrm{i})
\end{aligned}
$$

In order to show that the converse direction is in general not true we consider the type $\tau=(2)$. Further let $\beta: W_{\tau}(X) \longrightarrow \mathbb{N}$ be a bijection. (Such a bijection exists since $W_{\tau}(X)$ is countable.) For $t \in W_{(2)}(X)$ we define $\alpha_{t}: \operatorname{add}(t) \longrightarrow \mathbb{N}$ with $\alpha_{t}(a)=\beta(t)$ for all $a \in \operatorname{add}(t)$. Then $C:=\left\{\alpha_{t} \mid t \in W_{(2)}(X)\right\}$ is a coloration of $W_{(2)}(X)$.

Let $u \approx v \in W_{\tau}(X)^{2}$. If $u=v$ then obviously $\chi_{C}^{E}[u \approx v] \subseteq\{w \approx$ $\left.w \mid w \in W_{(2)}(X)\right\} \subseteq I d V_{H S}$. If $u \neq v$ and $u, v \in X$ then we get $\chi_{C}^{E}[u \approx v]=\{u \approx v\} \nsubseteq I d V_{H S}$. If $u \neq v$ and $u \notin X$ then $\beta(u) \neq \beta(v)$ and we may consider the multi-hypersubstitution $\rho \in \operatorname{Hyp}(2)^{\mathbb{N}}$ with $\rho(\beta(u))=\sigma_{x y}$ and $\rho(\beta(v))=\sigma_{x}$. Then $\widehat{\rho}_{C}[u]=u$ and $\widehat{\rho}_{C}[v]=r$, where $r$ is the first letter in $v$. Thus $\widehat{\rho}_{C}[u] \approx \widehat{\rho}_{C}[v] \notin I d V_{H S}$, i.e. $\chi_{C}^{E}[u \approx v] \nsubseteq$ $I d V_{H S}$. If $u \neq v$ and $v \notin X$ then we get $\chi_{C}^{E}[u \approx v] \nsubseteq I d V_{H S}$ in the dual manner. Altogether we have $\mathcal{C} I d V_{H S}=\left\{w \approx w \mid w \in W_{(2)}(X)\right\}$ and consequently, $\mathcal{C} M o d \mathcal{C} I d V_{H S}=\operatorname{Mod}\left\{w \approx w \mid w \in W_{(2)}(X)\right\}=A \lg (2)$ by Lemma 3.10 (i) and Proposition 3.13 (i).

On the other hand we have

$$
\operatorname{ModId} \chi_{C}^{A}\left[V_{H S}\right]=\operatorname{ModId} \chi^{A}\left[V_{H S}\right]=\operatorname{ModId} V_{H S}=V_{H S}
$$

by Lemma 3.6 and since $V_{H S}$ is solid. This shows that

$$
\operatorname{ModId} \chi_{C}^{A}\left[V_{H S}\right] \neq \mathcal{C} M o d \mathcal{C} I d V_{H S} \text {. }
$$

(ii) We have

$$
\mathcal{C} I d \mathcal{C} M o d \Sigma \subseteq \operatorname{Id} \chi_{C}^{A}[\mathcal{C} M o d \Sigma]=\operatorname{IdCMod} \Sigma=\operatorname{IdMod} \chi_{C}^{E}[\Sigma]
$$

by Proposition 3.13 (ii), Lemma 3.10 (ii) and Proposition 3.13 (i).

In order to show that the converse direction is in general not true we consider the type (2) and the coloration $C$ of $W_{(2)}(X)$ from Example 
3.11. Moreover let $\Sigma \subseteq W_{\tau}(X)^{2}$ be defined as in Example 3.11. Then we have $\mathcal{C} I d \mathcal{C} M$ od $\Sigma=\mathcal{C} I d V_{H S}=\Sigma$.

On the other hand we have $\operatorname{IdMod} \chi_{C}^{E}[\Sigma]=\operatorname{IdCMod} \Sigma=\operatorname{Id} V_{H S} \neq \Sigma$ by Proposition 3.13 (i). Consequently, $\mathcal{C} \operatorname{IdC} \operatorname{Mod} \Sigma \neq \operatorname{IdMod} \chi_{C}^{E}[\Sigma]$.

\section{C-COlored hyPEREQUATIONAL THEORIES}

Definition 4.1. Let $\Sigma$ be an equational theory of type $\tau$. Then $\Sigma$ is said to be a $C$-colored hyperequational theory if $\mathcal{C} \operatorname{Mod} \Sigma=\operatorname{Mod} \Sigma$.

$C$-colored hyperequational theories can be characterized in the same way as usual hyperequational theories:

Theorem 4.2. Let $\Sigma \in \mathcal{E}(\tau)$. Then the following statements are equivalent:

(i) $\mathcal{C} \operatorname{Mod} \Sigma=\operatorname{Mod} \Sigma$.

(ii) $\Sigma=\mathcal{C} I d \mathcal{C} M o d \Sigma$.

(iii) $\Sigma=\chi_{C}^{E}[\Sigma]$.

(iv) $\mathcal{C} I d M o d \Sigma=\Sigma$.

Proof: (i) $\Longrightarrow$ (ii): Since $\mathcal{C} I d \mathcal{C} M$ od is a closure operator we have $\Sigma \subseteq$ $\mathcal{C} I d \mathcal{C} M o d \Sigma$. Conversely, $\mathcal{C} I d \mathcal{C} M o d \Sigma \subseteq \operatorname{IdCMod} \Sigma=\operatorname{IdMod} \Sigma=\Sigma$.

(ii) $\Longrightarrow$ (iii): Clearly, $\Sigma \subseteq \chi_{C}^{E}[\Sigma]$. Conversely, let $u \approx v \in \chi_{C}^{E}[\Sigma]=$ $\chi_{C}^{E}[\mathcal{C} \operatorname{IdC} \operatorname{Mod} \Sigma]$ (because of (ii)). Then there is an $s \approx t \in \mathcal{C} \operatorname{Id\mathcal {C}Mod} \Sigma$ with $u \approx v \in \chi_{C}^{E}[s \approx t]$. Because of $s \approx t \in \mathcal{C} \operatorname{IdC} \operatorname{Mod} \Sigma$ we have $\chi_{C}^{E}$ $[s \approx t] \subseteq \operatorname{IdCMod} \Sigma$. With

$$
K:=\mathcal{C} \operatorname{Mod} \Sigma \text { and } \mathcal{C} I d K=\mathcal{C} I d \mathcal{C} M o d \Sigma=\Sigma
$$

by Proposition 3.8 we get $K=\operatorname{Mod} \Sigma$. Thus $\operatorname{IdCMod} \Sigma=\operatorname{Id} \operatorname{Mod} \Sigma=$ $\Sigma$ since $\Sigma \in \mathcal{E}(\tau)$. Altogether we have

$$
u \approx v \in \chi_{C}^{E}[s \approx t] \subseteq \operatorname{IdCMod} \Sigma=\Sigma .
$$

This shows that $\chi_{C}^{E}[\Sigma] \subseteq \Sigma$.

(iii) $\Longrightarrow$ (i): $\mathcal{C} \operatorname{Mod} \Sigma \subseteq \operatorname{Mod} \Sigma$ is clear. Conversely, let $\mathcal{A} \in \operatorname{Mod} \Sigma$. Then $\Sigma \subseteq I d \mathcal{A}$. Because of (iii) we have then $\chi_{C}^{E}[\Sigma] \subseteq I d \mathcal{A}$, i.e. $\mathcal{A} \in \mathcal{C} M o d \Sigma$. This shows that $\operatorname{Mod} \Sigma \subseteq \mathcal{C} \operatorname{Mod} \Sigma$.

(i) $\Leftrightarrow$ (iv): Suppose that $\mathcal{C} \operatorname{Id} \operatorname{Mod} \Sigma=\Sigma$. Then we put

$$
K:=\mathcal{C} \operatorname{ModC} I d \operatorname{Mod} \Sigma .
$$

Now we have

$$
\mathcal{C} M o d \Sigma=\mathcal{C} M o d \mathcal{C} I d M o d \Sigma=K
$$

and

$$
\mathcal{C} I d K=\mathcal{C} I d \mathcal{C} M o d \mathcal{C} I d M o d \Sigma=\mathcal{C} I d M o d \Sigma
$$


(since $(\mathcal{C} I d, \mathcal{C} M o d)$ is a Galois-connection). By Proposition 3.8 we get $K=\operatorname{Mod} \Sigma$, i.e. $\mathcal{C} M o d C I d M o d \Sigma=\operatorname{Mod} \Sigma, \mathcal{C} M o d \Sigma=\operatorname{Mod} \Sigma$ and $\Sigma$ is a $C$-colored hyperequational theory.

Suppose that $\Sigma$ is a $C$-colored hyperequational theory. We use that $\Sigma=\operatorname{IdMod} \Sigma \supseteq \mathcal{C} I d M o d \Sigma$. Now let $u \approx v \in \Sigma$. Assume that $u \approx$ $v \notin \mathcal{C} I d M o d \Sigma$. Then $\chi_{C}^{E}[u \approx v] \nsubseteq I d M o d \Sigma$ and in particular there is an $\mathcal{A} \in \operatorname{Mod} \Sigma$ with $\chi_{C}^{E}[u \approx v] \nsubseteq I d \mathcal{A}$. Since $\Sigma$ is a $C$-colored hyperequational theory we have $\operatorname{Mod} \Sigma=\mathcal{C} M o d \Sigma$, i.e. $\mathcal{A} \in \mathcal{C} M o d \Sigma$ and $\chi_{C}^{E}[\Sigma] \subseteq I d \mathcal{A}$. Because of $u \approx v \in \Sigma$ we have $\chi_{C}^{E}[u \approx v] \subseteq \chi_{C}^{E}[\Sigma]$ and thus $\chi_{C}^{E}[u \approx v] \subseteq I d \mathcal{A}$, a contradiction. Consequently, $u \approx v \in$ $\mathcal{C} I d M o d \Sigma$. This shows that $\Sigma \subseteq \mathcal{C} I d M o d \Sigma$.

When $\Sigma$ is a $C$ - colored hyperequational theory, then $\operatorname{Mod} \Sigma=$ $\chi_{C}^{A}[\operatorname{Mod} \Sigma]$. To see this, suppose that $\operatorname{Mod} \Sigma \neq \chi_{C}^{A}[\operatorname{Mod} \Sigma]$, then $\operatorname{Mod} \Sigma \neq \chi^{A}[\operatorname{Mod} \Sigma]$ by Lemma 3.6, i.e. $\operatorname{Mod} \Sigma$ is not solid. Then there is an $\mathcal{A} \in \operatorname{Mod} \Sigma$ with $\chi^{E}[\Sigma] \not I I d \mathcal{A}$. Because of $\chi^{E}[\Sigma] \subseteq \chi_{C}^{E}[\Sigma]$ we have $\chi_{C}^{E}[\Sigma] \nsubseteq I d \mathcal{A}$, i.e. $\mathcal{A} \notin \mathcal{C} M o d \Sigma$ contradicts $\mathcal{C} M o d \Sigma=\operatorname{Mod} \Sigma$.

The following example shows that the converse is not true, i.e. $C$ colored hyperequational theories cannot be characterized by the condition $\operatorname{Mod} \Sigma=\chi_{C}^{E}[\operatorname{Mod} \Sigma]$.

Example 4.3. Let $\tau=(2)$. We use the coloration $C$ from Example 3.11. Further we take $\Sigma:=I d V_{H S}$. Then we have $\operatorname{ModI} I V_{H S}=$ $V_{H S}=\chi^{A}\left[V_{H S}\right]$, since $V_{H S}$ is solid. By Lemma 3.6 we have $V_{H S}=\chi_{C}^{A}$ $\left[V_{H S}\right]=\chi_{C}^{A}\left[\operatorname{ModI} I V_{H S}\right]$.

Now we show that $\mathcal{C} M o d I d V_{H S} \neq V_{H S}=\operatorname{ModI} d V_{H S}$. Actually we will show that $\mathcal{C} M o d I d V_{H S} \subseteq \mathbf{B}$, where $\mathbf{B}$ denotes the variety of bands. We consider the term $s$ and the hypersubstitution $\rho$ from Example 3.11 and note that the application of $\rho$ to the identity $s \approx f(x, x) \in$ $I d V_{H S}$ provides the idempotent law. Thus $x \approx x^{2} \in \chi_{C}^{E}\left[I d V_{H S}\right]$. Let $\mathcal{A} \in \mathcal{C} M o d I d V_{H S}$. Then $\chi_{C}^{E}\left[I d V_{H S}\right] \subseteq I d \mathcal{A}$, and in particular $x \approx x^{2} \in \operatorname{Id} \mathcal{A}$, i.e. $\mathcal{A} \in \mathbf{B}$.

\section{Characterizations of COlORED SOlid Varieties}

In Section 3 colored solid varieties $V$ were defined by the property $I d V=\chi_{C}^{E}[I d V]$. We get the following characterization:

Theorem 5.1. Let $C$ be a coloration of $W_{\tau}(X)$ and $V$ be a variety of type $\tau$. Then the following statements (i)-(iii) are equivalent:

(i) $\mathcal{C} M o d I d V=V$.

(ii) $I d V=\mathcal{C} I d V$. 
(iii) $\chi_{C}^{E}[I d V]=I d V$.

Further, each of the statements (i)-(iii) implies both $V=\mathcal{C} M o d \mathcal{C} I d V$ and $\chi_{C}^{A}[V]=V$, but the converse implications are in general not true.

Proof: (i) $\Rightarrow$ (ii) Suppose that $\mathcal{C} M o d I d V=V$. Then $I d V$ is a $C$-colored hyperequational theory, i.e. $\operatorname{Mod} I d V=\mathcal{C} M o d I d V$ and $\mathcal{C} I d \mathcal{C} M o d I d V=I d V$. Thus $\mathcal{C} I d V=I d V$, i.e. $V$ is $C$-colored solid.

(ii) $\Rightarrow$ (iii) Since $\chi_{C}^{E}$ is a closure operator we have $I d V \subseteq \chi_{C}^{E}[I d V]$. Conversely, let $u \approx v \in \chi_{C}^{E}[I d V]$. Then there is an $s \approx t \in I d V$ with $u \approx v \in \chi_{C}^{E}[s \approx t]$. From $s \approx t \in I d V$ it follows that $s \approx t \in \mathcal{C} I d V$ by (ii), i.e. $\chi_{C}^{E}[s \approx t] \subseteq I d V$. Altogether we have $u \approx v \in I d V$. This shows that $\chi_{C}^{E}[I d V] \subseteq I d V$.

(iii) $\Rightarrow$ (i) $I d V$ is an equational theory. Thus we can use Theorem 4.2 and from $I d V=\chi_{C}^{E}[I d V]$ it follows that $\mathcal{C} M o d I d V=\operatorname{Mod} I d V$ and $I d V=\mathcal{C} I d \mathcal{C} M o d I d V$. This gives

$$
I d V=\mathcal{C} I d \mathcal{C} M o d I d V=\mathcal{C} I d M o d I d V=\mathcal{C} I d V .
$$

Moreover we have

$$
V \subseteq \mathcal{C} M o d \mathcal{C} I d V=\mathcal{C} M o d I d V=\operatorname{Mod} I d V=V .
$$

This shows that $V=\mathcal{C} M o d I d V$.

Suppose that $I d V=\mathcal{C} I d V$. We show that $V=\mathcal{C} M o d \mathcal{C} I d V$. First we have that $V \subseteq \mathcal{C} M o d \mathcal{C} I d V$ since $\mathcal{C} M o d \mathcal{C} I d$ is a closure operator. Conversely we get that $\mathcal{C} M o d I d V \subseteq M o d I d V=V$. Using $I d V=$ $\mathcal{C} I d V$ one obtains $\mathcal{C} M$ odC $I d V \subseteq V$.

Now we show that $V=\chi_{C}^{A}[V]$. Assume that $V \neq \chi_{C}^{A}[V]$. Then $V \neq \chi^{A}[V]$ by Lemma 3.6, i.e. $V$ is not solid and $\chi^{E}[I d V] \nsubseteq I d V$. Because of $\chi^{E}[I d V] \subseteq \chi_{C}^{E}[I d V]$ we have that $\chi_{C}^{E}[I d V] \nsubseteq I d V$. This shows that $I d V \nsubseteq \mathcal{C} I d V$, contradicting $I d V=\mathcal{C} I d V$.

Finally we prove that the opposite implication is not satisfied. Let $\tau=(2)$. Then there are a coloration $C$ of $W_{(2)}(X)$, a variety $V$ of type (2) with $V=\mathcal{C} M o d \mathcal{C} I d V$ and $V=\chi_{C}^{A}[V]$ such that $\mathcal{C} I d V \neq I d V$. Indeed, we take the coloration $C$ and the set $\Sigma$ from the proof of Example 3.11. There we have shown that $\mathcal{C} M o d \Sigma=V_{H S}$ and $\mathcal{C} I d V_{H S}=\Sigma$. So we have $V_{H S}=\mathcal{C} M o d \Sigma=\mathcal{C} M o d \mathcal{C} I d V_{H S}$. On the other hand there holds $V_{H S}=\chi^{A}\left[V_{H S}\right]$ since $V_{H S}$ is solid. Thus $V_{H S}=\chi_{C}^{A}\left[V_{H S}\right]$ by Lemma 3.6. Moreover there holds $\mathcal{C} I d V_{H S}=\Sigma \neq I d V_{H S}$.

$C$-colored hyperequational theories also have the following properties. 
Proposition 5.2. Let $C$ be a coloration of $W_{\tau}(X)$ and $\Sigma$ be an equational theory. If IdCMod $\Sigma=\Sigma$ then $\Sigma$ is a $C$-colored hyperequational theory. The converse direction is not true.

Proof: Suppose that $\operatorname{IdCMod} \Sigma=\Sigma$. Clearly, $\Sigma \subseteq \mathcal{C} I d \mathcal{C} M o d \Sigma$. Let $u \approx v \in \mathcal{C} I d \mathcal{C} M o d \Sigma$. Then $\chi_{C}^{E}[u \approx v] \subseteq \operatorname{IdCMod} \Sigma$, i.e. $u \approx v \in$ $\chi_{C}^{E}[u \approx v] \subseteq \operatorname{IdCMod} \Sigma=\Sigma$ and $u \approx v \in \Sigma$. This shows $\mathcal{C} I d \mathcal{C} M o d \Sigma \subseteq$ $\Sigma$. Altogether we have $\Sigma=\mathcal{C} I d \mathcal{C} M o d \Sigma$. By Theorem 4.2 we have $\mathcal{C} M o d \Sigma=\operatorname{Mod} \Sigma$, i.e. $\Sigma$ is a $C$-colored hyperequational theory.

In order to show that the converse direction is not true we consider the type (2) and the coloration $C$ of $W_{(2)}(X)$ from Example 3.11. Moreover let $\Sigma \subseteq W_{\tau}(X)^{2}$ be defined as in Example 3.11. Then we have $\Sigma=\mathcal{C} I d V_{H S}=\mathcal{C} I d \mathcal{C} M o d \Sigma$, i.e. $\Sigma$ is a $C$-colored hyperequational theory. But there holds $I d \mathcal{C} M o d \Sigma=I d V_{H S} \neq \Sigma$.

Proposition 5.3. Let $C$ be a coloration of $W_{\tau}(X)$ and $K$ be a class of algebras of type $\tau$. Then ModCIdK $=\mathcal{C} M o d C I d K$.

Proof: We have $\mathcal{C} M o d \mathcal{C} I d K=\left\{\mathcal{A} \mid \mathcal{A} \in A \lg (\tau), \chi_{C}^{E}[\mathcal{C} I d K] \subseteq\right.$ $I d \mathcal{A}\}=\{\mathcal{A} \mid \mathcal{A} \in A \lg (\tau), \mathcal{C} I d K \subseteq I d \mathcal{A}\}=\operatorname{ModC} I d K$ by Lemma 3.10 (i).

\section{EXAMPLES}

For a variety to be $C$-colored solid, it must satisfy all identities obtained by applying all multi-hypersubstitutions to all identities of the variety. This can be difficult to verify. In the hyperidentity case, if we want to check whether a variety of the form $V=\operatorname{Mod} \Sigma$ is solid, we have to apply the hypersubstitutions only to the set $\Sigma$. This is based on the following theorem:

Theorem 6.1. 1] Let $K \subseteq A \lg (\tau)$ and $\Sigma \subseteq W_{\tau}(X)^{2}$ and let $\mathcal{M} \subseteq$ $\mathcal{H} y p(\tau)$ be a submonoid. Then the following holds:

(i) $\chi_{M}^{E}[\Sigma] \subseteq \operatorname{IdMod} \Sigma \Longleftrightarrow \operatorname{IdMod} \Sigma=H_{M} I d H_{M} \operatorname{Mod} \Sigma$.

(ii) $\chi_{M}^{E}[\Sigma] \subseteq \operatorname{IdMod} \Sigma \Longleftrightarrow \chi_{M}^{E}[\operatorname{IdMod} \Sigma] \subseteq \operatorname{IdMod} \Sigma$.

(iii) $\chi_{M}^{A}[K] \subseteq \operatorname{ModIdK} \Longleftrightarrow \operatorname{ModIdK}=H_{M} M o d H_{M} I d K$.

(iv) $\chi_{M}^{A}[K] \subseteq \operatorname{ModI} d K \Longleftrightarrow \chi_{M}^{A}[\operatorname{ModIdK} \subseteq \operatorname{ModIdK}$.

For multi-hypersubstitutions and colored terms we have:

Theorem 6.2. Let $K \subseteq A \lg (\tau)$, let $C$ be a coloration of $W_{\tau}(X)$ and $\Sigma \subseteq W_{\tau}(X)^{2}$. Then the following holds:

(i) $\chi_{C}^{E}[\Sigma] \subseteq \operatorname{IdMod} \Sigma \Longleftrightarrow \operatorname{IdMod} \Sigma \supseteq \mathcal{C} I d \mathcal{C} M o d \Sigma$.

(ii) $\chi_{C}^{E}[\Sigma] \subseteq \operatorname{IdMod} \Sigma \Longleftarrow \chi_{C}^{E}[\operatorname{IdMod} \Sigma] \subseteq \operatorname{IdMod} \Sigma$.

(iii) $\chi_{C}^{A}[K] \subseteq \operatorname{ModIdK} \Longleftarrow \operatorname{ModIdK}=\mathcal{C} M o d C I d K$. 
(iv) $\chi_{C}^{A}[K] \subseteq M o d I d K \Longleftrightarrow \chi_{C}^{A}[\operatorname{ModIdK}] \subseteq \operatorname{ModIdK}$.

The converse implications are in general not satisfied.

Proof: (i): Suppose that $\chi_{C}^{E}[\Sigma] \subseteq \operatorname{IdMod\Sigma }$. Then we have

$$
\mathcal{C} \operatorname{IdCMod} \Sigma \subseteq \operatorname{IdMod} \chi_{C}^{E}[\Sigma] \subseteq \operatorname{IdModIdMod} \Sigma=\operatorname{IdMod} \Sigma
$$

by Proposition 3.15 (i).

Suppose that $\operatorname{IdMod} \Sigma \supseteq \mathcal{C} \operatorname{IdC} \operatorname{Mod} \Sigma$. Then we have

$$
\chi_{C}^{E}[\Sigma] \subseteq \mathcal{C} \operatorname{IdC} \operatorname{Mod} \chi_{C}^{E}[\Sigma]=\mathcal{C} \operatorname{Id} \operatorname{Cod} \Sigma \subseteq \operatorname{IdMod} \Sigma
$$

because of $\mathcal{C} M o d \Sigma=\operatorname{Mod} \chi_{C}^{E}[\Sigma]=\operatorname{Mod} \chi_{C}^{E}\left[\chi_{C}^{E}[\Sigma]\right]=\mathcal{C} \operatorname{Mod} \chi_{C}^{E}[\Sigma]$ (Proposition 3.13).

In order to show that the implication

$$
\chi_{C}^{E}[\Sigma] \subseteq \operatorname{IdMod} \Sigma \Longrightarrow \operatorname{IdMod} \Sigma=\mathcal{C} \operatorname{IdC} \operatorname{Mod} \Sigma
$$

is in general not true we consider the type (2) and the coloration $C$ of $W_{(2)}(X)$ from Example 3.11. Moreover let $\Sigma \subseteq W_{\tau}(X)^{2}$ be defined as in Example 3.11, Then we have

$$
\chi_{C}^{E}\left[\chi_{C}^{E}[\Sigma]\right]=\chi_{C}^{E}[\Sigma] \subseteq \operatorname{IdMod} \chi_{C}^{E}[\Sigma] .
$$

On the other hand we have

$$
\mathcal{C} I d \mathcal{C} M o d \chi_{C}^{E}[\Sigma]=\mathcal{C} I d \mathcal{C} M o d \Sigma=\mathcal{C} I d V_{H S}=\Sigma
$$

and

$$
\operatorname{IdMod} \chi_{C}^{E}[\Sigma]=\operatorname{IdC} \operatorname{Mod} \Sigma=I d V_{H S} \neq \Sigma .
$$

This shows that indeed $\chi_{C}^{E}\left[\chi_{C}^{E}[\Sigma]\right] \subseteq \operatorname{IdMod} \chi_{C}^{E}[\Sigma]$ but $\operatorname{IdMod} \chi_{C}^{E}[\Sigma] \neq$ $\mathcal{C} I d \mathcal{C} M$ od $\chi_{C}^{E}[\Sigma]$

(ii): This implication is clear since $\chi_{C}^{E}[\Sigma] \subseteq \chi_{C}^{E}[\operatorname{Id} \operatorname{Mod} \Sigma]$.

In order to show that the converse direction is in general not true we consider the type (2) and the coloration $C$ of $W_{(2)}(X)^{2}$ from Example 3.11. Moreover let $\Sigma \subseteq W_{\tau}(X)$ be defined as in Example 3.11. Then we have $\chi_{C}^{E}\left[\chi_{C}^{E}[\Sigma]\right]=\chi_{C}^{E}[\Sigma] \subseteq \operatorname{IdMod} \chi_{C}^{E}[\Sigma]$.

On the other hand we have $\operatorname{IdMod} \chi_{C}^{E}[\Sigma]=I d V_{H S}$. Further we have $x \approx x^{2} \in \chi_{C}^{E}\left[I d V_{H S}\right]$. Since $x \approx x^{2} \notin I d V_{H S}$ we have $\chi_{C}^{E}\left[I d V_{H S}\right] \nsubseteq$ $I d V_{H S}$ and thus

$$
\chi_{C}^{E}\left[\operatorname{IdMod} \chi_{C}^{E}[\Sigma]\right]=\chi_{C}^{E}\left[\operatorname{Id} V_{H S}\right] \nsubseteq I d V_{H S}=\operatorname{IdMod} \chi_{C}^{E}[\Sigma] .
$$

(iii): Suppose that $\operatorname{ModIdK}=\mathcal{C} M o d \mathcal{C} I d K$. Then we have $\chi_{C}^{A}[K] \subseteq$ $\operatorname{ModId} \chi_{C}^{A}[K] \subseteq \mathcal{C} M o d \mathcal{C} I d K=\operatorname{ModIdK}$ by Proposition 3.15 (i).

In order to show that the converse implication is in general not true we consider the type (2) and the coloration $C$ of $W_{(2)}(X)$ from Example 3.11. Then we have $\chi_{C}^{A}\left[V_{H S}\right]=V_{H S} \subseteq \operatorname{Mod} I d V_{H S}$. 
On the other hand we have

$$
\begin{gathered}
\mathcal{C} M o d \mathcal{C} I d V_{H S}=\operatorname{ModC} I d V_{H S} \\
=\operatorname{Mod}\left\{w \approx w \mid w \in W_{(2)}(X)\right\}=A \lg (2) \neq V_{H S}=\operatorname{ModI} I V_{H S}
\end{gathered}
$$

(see proof of Proposition 3.15 (i)).

(iv): is clear.

The following example shows once more that $\left(\chi_{C}^{E}, \chi_{C}^{A}\right)$ does not form a conjugate pair.

Proposition 6.3. If $\tau=\left(n_{i}\right)_{i \in I}$ with $n_{k} \geq 2$ for some $k \in I$ then there are a coloration $C$ of $W_{\tau}(X)$, terms $s, t \in W_{\tau}(X)$, and a multihypersubstitution $\rho \in \operatorname{Hyp}(\tau)^{\mathbb{N}}$ and an algebra $\mathcal{A}$ of type $\tau$ such that

$$
\mathcal{A} \models \widehat{\rho}_{C}[s] \approx \widehat{\rho}_{C}[t] \Longleftrightarrow \rho[\mathcal{A}] \models s \approx t
$$

is not valid.

Proof: We will show that for the free algebra $\mathcal{F}_{\tau}(X)$ of type $\tau$ over an alphabet $X$ there are a coloration $C$ of $W_{\tau}(X)$ and some $s, t \in$ $W_{\tau}(X)$ and an $\rho \in \operatorname{Hyp}(\tau)^{\mathbb{N}}$ such that $\widehat{\rho}[s] \approx \widehat{\rho}[t] \in \operatorname{IdF}_{\tau}(X)$ but $s \approx t \notin I d \rho\left[\mathcal{F}_{\tau}(X)\right]$.

Let $k \in I$ with $n_{k} \geq 2$. We put $s:=f_{k}\left(f_{k}\left(x_{1}, \ldots, x_{1}\right), x_{2}, \ldots, x_{2}\right)$ and $t:=f_{k}\left(f_{k}\left(x_{1}, \ldots, x_{1}\right), x_{1}, \ldots, x_{1}\right)$ and we consider a coloration $C$ of $W_{\tau}(X)$ with the following properties:

(i) $\alpha_{s}(j)=0$ for all $j \in \operatorname{add}(s)$,

(ii) $\alpha_{t}(j)=0$ for all $j \in \operatorname{add}(t)$,

(iii) $\alpha_{f_{k}\left(x_{1}, \ldots, x_{n_{k}}\right)}(j)=1$ for all $j \in \operatorname{add}\left(f_{k}\left(x_{1}, \ldots, x_{n_{k}}\right)\right)$.

Finally, let $\rho \in H y p^{\mathbb{N}}$ given by $\rho(0)=\sigma_{x_{1}}$ and $\rho(j)=\widetilde{\sigma}$ for $j \in \mathbb{N} \backslash\{0\}$, where $\sigma_{x_{1}}$ is defined by $\sigma_{x_{1}}: f_{j} \longrightarrow x_{1}$ for all $j \in I$ and $\widetilde{\sigma}$ is defined by $\widetilde{\sigma}: f_{j} \longrightarrow x_{n_{j}}$ for all $j \in I$.

Then we have $\widehat{\rho}_{C}[s]=\widehat{\rho}_{C}[t]=x_{1}$. This shows that $\widehat{\rho}_{C}[s] \approx \widehat{\rho}_{C}[t] \in$

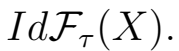

On the other hand we have

$$
f_{k}^{\rho\left[\mathcal{F}_{\tau}(X)\right]}=\widehat{\rho}_{C}\left[f_{k}\left(x_{1}, \ldots, x_{n_{k}}\right)\right]^{\mathcal{F}_{\tau}(X)}=x_{n_{k}}^{\mathcal{F}_{\tau}(X)} .
$$

Then the following holds:

$$
\begin{aligned}
s^{\rho\left[\mathcal{F}_{\tau}(X)\right]} & \left(x_{1}, x_{2}\right) \\
& =f_{k}^{\rho\left[\mathcal{F}_{\tau}(X)\right]}\left(f_{k}^{\rho\left[\mathcal{F}_{\tau}(X)\right]}\left(x_{1}, \ldots, x_{1}\right), x_{2}, \ldots, x_{2}\right) \\
& =x_{n_{k}}^{F_{\tau}(X)}\left(x_{n_{k}}^{\mathcal{F}_{\tau}(X)}\left(x_{1}, \ldots, x_{1}\right), x_{2}, \ldots, x_{2}\right) \\
& =x_{2}
\end{aligned}
$$


and

$$
\begin{aligned}
t^{\rho\left[\mathcal{F}_{\tau}(X)\right]} & \left(x_{1}, x_{2}\right) \\
& =f_{k}^{\rho\left[\mathcal{F}_{\tau}(X)\right]}\left(f_{k}^{\rho\left[\mathcal{F}_{\tau}(X)\right]}\left(x_{1}, \ldots, x_{1}\right), x_{1}, \ldots, x_{1}\right) \\
& =x_{n_{\tau}}^{\mathcal{F}_{\tau}(X)}\left(x_{n_{k}}^{\mathcal{F}_{\tau}(X)}\left(x_{1}, \ldots, x_{1}\right), x_{1}, \ldots, x_{1}\right) \\
& =x_{1} .
\end{aligned}
$$

This shows $s^{\rho\left[\mathcal{F}_{\tau}(X)\right]} \neq t^{\rho\left[\mathcal{F}_{\tau}(X)\right]}$ and $s \approx t \notin I d \rho\left[\mathcal{F}_{\tau}(X)\right]$.

Example 6.4. In the next example we will determine a collection of colorations $C$ of $W_{(2)}(X)$ such that all solid varieties of bands are $C$ colored solid. There are exactly three nontrivial solid varieties of bands: the variety $R B$ of all rectangular bands, the variety $N B$ of all normal bands, and the variety $\operatorname{Reg} B$ of all regular bands (see [4]).

We split the set $W_{(2)}(X)$ into two sets $A$ and $B$ :

$$
\begin{aligned}
& A:=\bigcup\left\{W_{(2)}(\{w\}) \mid w \in X\right\} \text { and } \\
& B:=W_{(2)}(X) \backslash A .
\end{aligned}
$$

By $\mathcal{F}$ we denote the set of all mappings

$$
\beta: \bigcup\{\{(t, a) \mid a \in \operatorname{add}(t)\} \mid t \in A\} \rightarrow \mathbb{N} .
$$

For $\beta \in \mathcal{F}$ we define a coloration $C_{\beta}=\left\{\alpha_{t}^{\beta} \mid t \in W_{(2)}(X)\right\}$ as follows: For $t \in W_{(2)}(X)$ and $a \in \operatorname{add}(t)$ we put

$$
\begin{array}{ll}
\alpha_{t}^{\beta}(a)=1 & \text { if } t \in B \\
\alpha_{t}^{\beta}(a)=\beta(t, a) & \text { if } t \in A
\end{array}
$$

Then the varieties $R B, N B$, and $\operatorname{Reg} B$ are $C_{\beta}$-colored solid. Indeed, let $V$ be one of the varieties $R B, N B$, and $\operatorname{Reg} B$ and let $s \approx t \in I d V$. Further let $\rho \in \operatorname{Hyp}(2)^{\mathbb{N}}$.

If $s, t \in A$ then there is a variable $w \in X$ such that $s, t \in W_{(2)}(\{w\})$. (Otherwise the identity $s \approx t$ provides the identity $x \approx y$ because of the associative and the idempotent law.) Thus $\widehat{\rho}_{C_{\beta}}[s], \widehat{\rho}_{C_{\beta}}[t] \in W_{(2)}(\{w\})$. Because of the associative and the idempotent law $\widehat{\rho}_{C_{\beta}}[s] \approx \widehat{\rho}_{C_{\beta}}[t]$ is an identity in $V$.

If $s, t \in B$ then $\widehat{\rho}_{C_{\beta}}[s]=\widehat{\rho(1)}[s]$ and $\widehat{\rho}_{C_{\beta}}[t]=\widehat{\rho(1)}[t]$ by Lemma 2.5. Since $V$ is solid, we have $\widehat{\rho(1)}[s] \approx \widehat{\rho(1)}[t] \in I d V$ and thus $\widehat{\rho}_{C_{\beta}}[s] \approx \widehat{\rho}_{C_{\beta}}[t] \in I d V$.

If $s \in A$ and $t \in B$ then there is a $w \in X$ such that $s \in W_{(2)}(\{w\})$. Since $V$ is a variety of bands we have $\widehat{\rho}_{C_{\beta}}[s] \approx w \approx \widehat{\rho(1)}[s]$. By Lemma 
2.5 we have $\widehat{\rho}_{C_{\beta}}[t]=\widehat{\rho(1)}[t]$, Since $V$ is solid we have $\widehat{\rho(1)}[s] \approx \widehat{\rho(1)}[t] \epsilon$ $I d V$ and thus $\widehat{\rho}_{C_{\beta}}[s] \approx \widehat{\rho}_{C_{\beta}}[t] \in I d V$.

If $s \in B$ and $t \in A$ then we get in a similar way that $\widehat{\rho}_{C_{\beta}}[s] \approx$ $\widehat{\rho}_{C_{\beta}}[t] \in I d V$.

Example 6.5. We consider the following coloration $C=\left\{\alpha_{t} \mid t \in\right.$ $\left.W_{(2)}(X)\right\}$ of $W_{(2)}(X)$ : for $t \in W_{(2)}(X)$ and $a \in \operatorname{add}(t)$ we put

$$
\begin{array}{ll}
\alpha_{t}(a)=1 & \text { if } t=f(x, x) ; \\
\alpha_{t}(a)=2 & \text { if } t \neq f(x, x) .
\end{array}
$$

The varieties $R B, N B$, and $\operatorname{Reg} B$ are the only nontrivial $C$-colored solid varieties of semigroups. Indeed, by Example 6.4, $R B, N B$, and $\operatorname{Reg} B$ are $C$-colored solid.

Conversely, let $V$ be a $C$-colored solid variety of semigroups and $\rho \in \operatorname{Hyp}(2)^{\mathbb{N}}$ with

$$
\rho(1)=\sigma_{x} \text { and } \rho(i)=\sigma_{x y} \text { for } 2 \leq i \in \mathbb{N} .
$$

Since $V$ is $C$-colored solid, $V$ is solid and thus $V \subseteq V_{H S}$, i.e. $f(x, x) \approx$ $f(f(x, x), f(x, x)) \in I d V$. Then we have:

$$
\begin{aligned}
x & \approx \widehat{\rho(1)}[f(x, x)] \approx \widehat{\rho}_{C}[f(x, x)] \text { (by Lemma 2.5 ) } \\
& \approx \widehat{\rho}_{C}[f(f(x, x), f(x, x))](V \text { is } C \text {-colored solid) } \\
& \approx \widehat{\rho}(2)[f(f(x, x), f(x, x))] \text { (by Lemma 2.5) } \\
& \approx f(f(x, x), f(x, x)) \\
& \approx f(x, x) .
\end{aligned}
$$

This shows that $V$ is a variety of bands. But $R B, N B$, and $\operatorname{Reg} B$ are the only nontrivial solid varieties of bands.

\section{REFERENCES}

[1] K. Denecke, M. Reichel, Monoids of hypersubstitutions and M-solid varieties, Contributions to General Algebra 9, Verlag B.G. Teubner, Stuttgart, 1995, 117-125.

[2] K. Denecke, S. L. Wismath, Hyperidentities and Clones, Gordon and Breach Science Publishers, 2000.

[3] K. Denecke, S. L. Wismath, Universal Algebra and Applications in Theoretical Computer Science, Chapman and Hall/ CRC, 2002.

[4] E. Graczýnska, On normal and regular identities and hyperidentities, Universal and Applied Algebra, Turawa, Poland 3 - 7 May 1988, World Scientific (1989), 107-135. 
[5] L. Polák, On hyperassociativity, Algebra Universalis 36 No. 3 (1996), 363-378.

[6] L. Polák, All solid varieties of semigroups, J. Algebra 219 No. 2 (1999), 421-436.

Authors' Addresses:

K. Denecke

Universität Potsdam

Fachbereich Mathematik

Postfach 601553

D-14415 Potsdam

email:kdenecke@rz. uni-potsdam.de

\section{J. Koppitz}

Universität Potsdam

Fachbereich Mathematik

Postfach 601553

D-14415 Potsdam

email:koppitz@rz.uni-potsdam.de

Sl. Shtrakov

South-West-University Blagoevgrad

Faculty of Mathematics and Natural Sciences

2700 Blagoevgrad, Bulgaria

e-mail:shtrakov@swu.bg 